\title{
Genetics of coronary artery calcification among African Americans, a meta-analysis
}

Mary K Wojczynski ${ }^{1 *}$, Mingyao Li ${ }^{2}$, Lawrence F Bielak ${ }^{3}$, Kathleen F Kerr ${ }^{4}$, Alex P Reiner ${ }^{5}$, Nathan D Wong ${ }^{6}$, Lisa R Yanek', Liming Qu ${ }^{2}$, Charles C White ${ }^{8}$, Leslie A Lange ${ }^{9}$, Jane F Ferguson ${ }^{10}$, Jing He ${ }^{2}$, Taylor Young ${ }^{11}$, Thomas H Mosley ${ }^{12}$, Jennifer A Smith ${ }^{3}$, Brian G Kral ${ }^{7}$, Xiuqing Guo ${ }^{13}$, Quenna Wong ${ }^{4}$, Santhi K Ganesh ${ }^{14}$, Susan R Heckbert ${ }^{5}$, Michael E Griswold ${ }^{15}$, Daniel H O'Leary ${ }^{16}$, Matthew Budoff ${ }^{17}$, J Jeffrey Carr ${ }^{18}$, Herman A Taylor, Jr. ${ }^{19,20}$, David A Bluemke ${ }^{21}$, Serkalem Demissie ${ }^{8}$, Shih-Jen Hwang ${ }^{22}$, Dina N Paltoo ${ }^{23}$, Joseph F Polak ${ }^{16}$, Bruce M Psaty ${ }^{24,25}$, Diane M Becker ${ }^{7}$, Michael A Province', Wendy S Post ${ }^{26}$, Christopher J O'Donnell ${ }^{27,28,29}$, James G Wilson ${ }^{30}$, Tamara B Harris ${ }^{31}$, Maryam Kavousi ${ }^{32,33}$, L Adrienne Cupples ${ }^{27,34}$, Jerome I Rotter ${ }^{13}$, Myriam Fornage ${ }^{35}$, Lewis C Becker ${ }^{7}$, Patricia A Peyser ${ }^{3}$, Ingrid B Borecki ${ }^{1 *+}$ and Muredach P Reilly ${ }^{10^{*}+}$

\begin{abstract}
Background: Coronary heart disease (CHD) is the major cause of death in the United States. Coronary artery calcification (CAC) scores are independent predictors of CHD. African Americans (AA) have higher rates of CHD but are less well-studied in genomic studies. We assembled the largest AA data resource currently available with measured CAC to identify associated genetic variants.

Methods: We analyzed log transformed CAC quantity $(\operatorname{In}(C A C+1))$, for association with $\sim 2.5$ million single nucleotide polymorphisms (SNPS) and performed an inverse-variance weighted meta-analysis on results for 5,823 AA from 8 studies. Heritability was calculated using family studies. The most significant SNPs among AAs were evaluated in European Ancestry (EA) CAC data; conversely, the significance of published SNPs for CAC/CHD in EA was queried within our AA meta-analysis.
\end{abstract}

Results: Heritability of CAC was lower in AA ( 30\%) than previously reported for EA ( $50 \%)$. No SNP reached genome wide significance ( $p<5 \mathrm{E}-08$ ). Of 67 SNPs with $p<1 \mathrm{E}-05$ in AA there was no evidence of association in EA CAC data. Four SNPs in regions previously implicated in CAC/CHD (at 9p21 and PHACTR1) in EA reached nominal significance for CAC in AA, with concordant direction. Among AA, rs16905644 ( $p=4.08 \mathrm{E}-05)$ had the strongest association in the 9p21 region.

Conclusions: While we observed substantial heritability for CAC in AA, we failed to identify loci for CAC at genome-wide significant levels despite having adequate power to detect alleles with moderate to large effects. Although suggestive signals in AA were apparent at 9p21 and additional CAC and CAD EA loci, overall the data suggest that even larger samples and an ethnic specific focus will be required for GWAS discoveries for CAC in AA populations.

Keywords: Atherosclerosis, Coronary artery calcium, Genetics, Meta-analysis, African-American

\footnotetext{
*Correspondence: mwojczynski@wustl.edu; iborecki@wustl.edu;

Muredach@mail.med.upenn.edu

${ }^{\dagger}$ Equal contributors

'Department of Genetics, Division of Statistical Genomics, Washington

University School of Medicine, St. Louis, MO, USA

${ }^{10}$ The Cardiovascular Institute and Department of Medicine, Perelman School

of Medicine, University of Pennsylvania, Philadelphia, PA, USA

Full list of author information is available at the end of the article
}

\section{Biomed Central}

(c) 2013 Wojczynski et al.; licensee BioMed Central Ltd. This is an Open Access article distributed under the terms of the Creative Commons Attribution License (http://creativecommons.org/licenses/by/2.0), which permits unrestricted use, distribution, and reproduction in any medium, provided the original work is properly cited. 


\section{Background}

Atherosclerotic coronary heart disease (CHD) is a complex heritable condition and the major cause of death in the United States [1]. Recent meta-analyses of genome wide association studies (GWAS) in individuals of European Ancestry (EA) have identified single nucleotide polymorphisms (SNPs) at over 30 independent regions associated with coronary artery disease (CAD) and myocardial infarction (MI) [2-6]; however, these loci explain less than 10\% of the heritability of the disease in EA. Although the burden of CHD is higher in African Americans (AA) than in EA [7-10], there are few contemporary genetic studies of CHD phenotypes in AA populations [11,12]. Moreover, adequately powered CHD GWAS in AA are lacking, with studies performed to date failing to identify any loci approaching genome wide significance [11]. The strongest loci for CAD/MI in EA GWAS [2-6], including the 9p21 locus, have shown inconsistent signals in small studies of AA [11] likely due to limited power and differences in linkage disequilibrium structure among the populations. Candidate gene studies of $\mathrm{CHD}$ in AA, however, have identified causal mutations that are private to AA populations [13].

One strategy for identifying genetic factors underlying susceptibility to CHD is to examine measures of subclinical atherosclerosis. Subclinical traits, such as coronary artery calcification $(\mathrm{CAC})$, provide quantitative measures with reduced heterogeneity compared to presence or absence of clinical disease. CAC quantity is associated with traditional and novel CHD risk factors, is directly related to the burden of coronary atherosclerosis on angiography as well as autopsy, and also predicts incident CHD events after controlling for risk factors [14-17]. CAC is heritable in populations of EA [18-21] with estimates ranging from 40-60\%. O'Donnell et al. [22] recently published the first large GWAS results $(n=9,992)$ of CAC in EAs which identified 49 SNPs in two distinct regions, 9p21 and the PHACTR1 gene on chromosome 6, surpassing genome wide significance $(\mathrm{p}<5 \mathrm{E}-08)$. Several of these SNPs were previously identified in EA GWAS of CAD/MI [2-6] providing support for CAC as a useful phenotype for discovery of novel CHD genes [22]. The lower prevalence of CAC in AA as compared to other ethnic groups, particularly persons of EA [23-25], might suggest, however, that their excess CHD rates may be attributed to differences in hypertension, diabetes, access to care, socioeconomic status or other CHD risk factors with limited influence on CAC $[23,26]$.

Here, we present the largest AA GWAS of CAC, including 5,823 AA individuals. Using meta-analysis, we interrogated the largest AA CAC dataset available to date with genome wide SNP genotypes obtained as part of study-specific projects or through the National Heart Lung and Blood Institute (NHLBI) Candidate gene
Association Resource (CARe) [27]. Our aims were to estimate the heritability of CAC in AA family samples, to perform a meta-analysis of GWAS results in an attempt to discover novel associations, and to assess the significance of genetic variants previously reported in subjects of EA.

\section{Methods}

\section{Ethics statement}

Each study obtained approval from their respective institutional review board and the ethics committee of each participating institution, including the University of Alabama at Birmingham, Washington University, University of Mississippi Medical Center, University of Minnesota, Northwestern University, Kaiser Permanente (Oakland, CA), University of Washington, Columbia University, Johns Hopkins School of Medicine, UCLA School of Medicine, Wake Forest University School of Medicine, University of Michigan Health Sciences and Behavioral Sciences, and the University of Pennsylvania. All participants gave written informed consent in accordance with institutional requirements and the principles expressed in the Declaration of Helsinki.

\section{Cohorts and CAC measurement}

Eight cohorts (total $\mathrm{N}$ of 5,823 ) of AA participants (Additional file 1: Supplemental Methods) with measures of CAC participated in the meta-analysis (Table 1, Family Heart Study (FamHS), $\mathrm{n}=596$; Jackson Heart Study (JHS), $\mathrm{n}=1,388$ (comprised of JHS de novo recruited sample "JHS", $\mathrm{n}=1066$ and a JHS sample previously enrolled in Atherosclerosis Risk in Communities (ARIC) study (“JHS-ARIC"), $\mathrm{n}=322$ ); Coronary Artery Risk Development In Young Adults (CARDIA), $\mathrm{n}=671$; Multi-Ethnic Study of Atherosclerosis (MESA), $n=1646$; MESA Family/Air, $\mathrm{n}=934$; Genetic Study of Atherosclerosis Risk (GeneSTAR), $\mathrm{n}=272$; and Genetic Epidemiology Network of Arteriopathy (GENOA), $\mathrm{n}=316$;). Four of these (JHS, JHS-ARIC, CARDIA, and MESA) were genotyped through the NHLBI CARe [27], while FamHS, MESA Family/Air, GeneSTAR, and GENOA subjects were genotyped separately through funding from NHLBI. Given the low prevalence of CAC in younger individuals, participants were excluded if they were $\leq 35$ years old. Participants were also excluded if they did not consent to genetic research or if genotype information did not meet cohortspecific quality-control standards (Additional file 1: Table S1 and Supplemental Methods). The definition of cardiovascular risk factors in each cohort is provided in the supplement. Participants provided written informed consent and protocols were approved by local institutional review boards.

All studies assessed CAC using computed tomography (CT, performed either by electron beam or multi-detector 
Table 1 Participant characteristics of eight participating African-American cohorts

\begin{tabular}{|c|c|c|c|c|c|c|c|c|}
\hline & \multirow[b]{2}{*}{ FamHS } & \multicolumn{4}{|c|}{ CARe cohorts } & \multirow[b]{2}{*}{ MESA Family/Air } & \multirow[b]{2}{*}{ GeneSTAR } & \multirow[b]{2}{*}{ GENOA } \\
\hline & & JHSt & CARDIA* & JHS-ARIC† & MESA & & & \\
\hline $\mathrm{N}$ analyzed & 596 & 1066 & 671 & 322 & 1646 & 934 & 272 & 316 \\
\hline CAC score $>0, n(\%)$ & $330(55.4 \%)$ & 419 (39.3\%) & $108(16.1 \%)$ & $224(69.6 \%)$ & $726(44.1 \%)$ & $388(41.8 \%)$ & $111(40.8 \%)$ & $214(67.7 \%)$ \\
\hline CAC score mean & 175.8 & 108.8 & 21.7 & 267.4 & 127.7 & 123.6 & 49.1 & 252.7 \\
\hline CAC score, median (Q1, Q3) & $0.8(0,73.3)$ & $0(0,37.8)$ & $0(0,0)$ & $41.5(0,260.7)$ & $0(0,53.2)$ & $0(0,40.2)$ & $0(0,16.0)$ & $37.4(0,266.6)$ \\
\hline CAC Heritability (SE) & $0.33(0.10)$ & $0.47(0.17)$ & - & - & - & $0.31(0.08) \neq$ & 0 (n/a) & $0.26(0.16)$ \\
\hline Age, mean (range) & $54.1(36-83)$ & $51.1(36-90)$ & $44.5(37-54)$ & $65.1(57-80)$ & $62.2(45-84)$ & 58.0 (39-91) & $51.2(36-64)$ & $69.6(56-85.5)$ \\
\hline Sex, $\mathrm{n}$ (\% male) & $202(33.9 \%)$ & $408(38.3 \%)$ & $244(36.4 \%)$ & $87(27 \%)$ & $745(45.3 \%)$ & $375(40.4 \%)$ & $92(33.8 \%)$ & $86(27.2 \%)$ \\
\hline Current Smoker, n (\%) & $144(24.2 \%)$ & $123(11.5 \%)$ & $157(23.5 \%)$ & $30(9.3 \%)$ & $297(18 \%)$ & $190(20.5 \%)$ & $75(27.6 \%)$ & $27(8.5 \%)$ \\
\hline Diabetic, n (\%) & $149(25.4 \%)$ & $145(13.6 \%)$ & $69(10.3 \%)$ & $77(23.9 \%)$ & $263(16 \%)$ & $137(14.8 \%)$ & $50(18.4 \%)$ & 105 (33.2\%) \\
\hline Hypertension, n (\%)§ & $458(76.9 \%)$ & $607(56.9 \%)$ & $229(34.1 \%)$ & $243(75.5 \%)$ & $981(59.6 \%)$ & $561(60.4 \%)$ & $186(68.4 \%)$ & $263(83.2 \%)$ \\
\hline Statin Users, n (\%)§ & $60(10.1 \%)$ & $95(8.9 \%)$ & $14(2.1 \%)$ & $53(16.5 \%)$ & $241(14.6 \%)$ & $193(20.8 \%)$ & $58(21.3 \%)$ & - \\
\hline Prevalent CHD, n (\%) & $67(11.2 \%)$ & $46(4.3 \%)$ & $13(1.9 \%)$ & $26(8.1 \%)$ & 0 & $46(4 . \%)$ & 0 & $7(2.2 \%)$ \\
\hline
\end{tabular}

*CARDIA: Year 20 data; hypertension by self-report.

† JHS CAC data comprised of the JHS de novo recruited sample "JHS" ( $n=1066)$ and the JHS sample previously enrolled in the ARIC study, denoted "JHS-ARIC" $(\mathrm{n}=322)$. CAC data were collected in all JHS participants (JHS and JHS-ARIC) through JHS NHLBI funding. The JHS-de novo recruited sample was genotyped as a batch via the CARe study at the Broad Institute. Genotyping of all AA ARIC participants also was performed as a separate batch via the CARe study at the Broad Institute. The recommendation from the CARe study analysis committee was to analyze the" JHS" and "JHS-ARIC" individuals separately because QC of JHS and ARIC genotype data was not $100 \%$ identical.

¥MESA Family/Air: heritability based on $n=882$ in families from participants of MESA Family.

$\S$ GeneSTAR: hypertension = average of 3 measures $\geq 140 / 90 \mathrm{mmHg}$ and/or current use of antihypertensive medication; GENOA: Self-reported hypertension. Statin use data was not available; MESA Family/Air: hypertension if diastolic > $=90$ or systolic > =140; or self-reported high blood pressure and on meds for hypertension; FamHS, CARDIA, MESA, JHS: hypertension = measure $\geq 140 / 90 \mathrm{~mm} \mathrm{Hg}$ and/or current use of antihypertensive medication.

CT) imaging methods (Additional file 1: Supplemental Methods). Scans were interpreted at the corresponding sites of the independent studies but all investigators applied standardized methods using published software and reading algorithms [28-30]. Calcified plaque was quantified by the Agatston method [31] and the total calcium score, summing over the individual coronary arteries (i.e. left main, left anterior descending, circumflex, and right coronary arteries), was used in these analyses. Each study performed quality control in obtaining CAC measurements. In order to maintain comparability to published results for samples of European descent [22], we used the identical phenotypic transformation, $\ln (\mathrm{CAC}+1)$, in our primary analysis. In secondary analyses, we assessed CAC dichotomously (present/absent), $\ln (\mathrm{CAC})$ for those with $\mathrm{CAC}>0$ and $\ln (\mathrm{CAC}+1)$ exclusively in the subset of older participants (men $\geq 50$ and women $\geq 60$ ). These secondary analyses produced similar results to the primary analysis and therefore these data are not presented.

\section{Power analysis}

We estimated the power of our accumulated study sample using the software QUANTO [32,33]. We specified a quantitative outcome with an effective number of independent subjects $\left(\mathrm{N}_{\mathrm{eff}}=5,186, \mathrm{~N}_{\text {total }}=5,823\right)$ accounting for the fact that several of the participating studies have family data, and gene only effect options, inputting sample size, estimated mean and standard deviation of $\ln$ $(\mathrm{CAC}+1)$, and assuming an additive genetic model. This approach also assumes that meta-analysis is equivalent to pooled analysis. We also varied the allelic frequency from 0.01 to 0.4 , and assessed three significance thresholds $0.05,1 \mathrm{E}-05$, and 5E-08 (two-sided). The effect size was characterized as $\mathrm{r}^{2}$, the proportion of phenotypic variance attributable to the SNP, which is a function of both allele frequency and the distance between genotype-specific means.

\section{Genotyping data and quality control}

The CARe genotyping center at the Broad Institute (for JHS, JHS-ARIC, CARDIA, and MESA) or each individual study (for FamHS, MESA Family/Air, GeneSTAR, and GENOA,) was responsible for quality control for the genotypes and imputation (details in Additional file 1: Supplemental Methods and summarized in Additional file 1: Table S1). All studies used $\mathrm{MaCH}$ [34] (http://www.sph. umich.edu/csg/abecasis/MaCH/) for imputation except MESA Family/Air which used Impute [35] software (http://mathgen.stats.ox.ac.uk/impute/impute.html). We report results for SNPs with coded allele frequency (CAF) between 1-99\%. More stringent CAF filters were used for MESA Family/Air $(5 \% \leq \mathrm{CAF} \leq 95 \%)$ and CARDIA $(10 \% \leq$ $\mathrm{CAF} \leq 90 \%$ ) due to small sample size, young mean age, and high prevalence of zero CAC which resulted in higher rates of Type I errors for SNPs with CAF less than 5 or $10 \%$ respectively; with these more stringent filters, the quantile-quantile (QQ) plots showed an acceptable fit (Additional file 2: Figure S1). SNPs with a 
Hardy-Weinberg equilibrium (HWE) test with $\mathrm{p}<1 \mathrm{E}-06$ were excluded, as were SNPs with a call rate $<0.95$ or SNPs with an imputation quality metric $\left(r^{2}\right)$ of less than 0.50 . In each study, hybrid datasets were created for analysis by substituting measured for imputed genotypes when available (Additional file 1: Supplemental Methods).

\section{Heritability calculation}

Five of the participating studies have family data (FamHS, JHS, MESA Family/Air (family component), GeneSTAR, GENOA) and a variance components model was used to obtain maximum likelihood estimates of polygenic heritability for the age, age ${ }^{2}$, sex, and principal components (only those deemed necessary for each study to characterize population stratification, Additional file 1: Table S1, as estimated by EIGENSTRAT [36]) adjusted residuals of $\ln (C A C+1)$ using the Sequential Oligogenic Linkage Analysis Routines (SOLAR) [37] software package.

\section{Cohort-specific analyses}

For each measured or imputed SNP, each cohort provided estimated regression coefficients and standard errors (SE), the identity of the coded allele, its frequency (CAF), and $\mathrm{p}$ for a linear regression model of $\ln (\mathrm{CAC}+1)$ on allelic dosage for each SNP, using an additive genetic model. Each cohort adjusted the analysis for the effects of age, age ${ }^{2}$, sex, age ${ }^{*}$ sex, age ${ }^{2 *}$ sex, CT scanner as needed, study site as needed, and the principal components deemed necessary for their study to characterize population stratification estimated by EIGENSTRAT [36] (Additional file 1: Table S1). A linear mixed effects model or Generalized Estimating Equation was used to account for correlation among participants in families.

\section{Meta-analyses}

An inverse variance-weighted meta-analysis with fixed effects was used to estimate summary effects (METAL software, http://www.sph.umich.edu/csg/abecasis/metal/ index.html) for the association of allelic dosage at each SNP with CAC $(n=5,823)$. Meta-analyses were performed independently at two sites (Washington University and University of Pennsylvania) for quality assurance and the results were concordant. Heterogeneity among studies was assessed using a $X^{2}$ test, and there was no significant heterogeneity for CAC quantity in our main results. We considered genome-wide significance as $\mathrm{p}<5 \mathrm{E}-08$, suggestive significance as $\mathrm{p}<1 \mathrm{E}-05$, and nominal significance as $\mathrm{p} \leq 0.05$. All tests were two-sided. SNPs reaching suggestive significance were assessed for their association with CAC in the EA CAC GWAS [22]. Conversely, we assessed the significance in our AA CAC meta-analysis data of EA CAC GWAS SNPs, including a close interrogation of both the genome wide significant loci for CAC in EA data [22]: the 9p21 region [11] and PHACTR1 locus.

\section{Results}

\section{Sample characteristics and heritability}

A description of each cohort is provided in the Additional file 1: Supplemental Methods. Demographic and selected risk factor characteristics of the 5,823 study participants are described in Table 1 by cohort. Gender distribution was similar across studies. There was, however, variation in the age range across cohorts, e.g., CARDIA (37-54 yrs) vs. GENOA (56-86 yrs), as well as some risk factors such as a higher prevalence of diabetes and hypertension in GENOA and more current smokers in GeneSTAR. Additional file 1: Supplemental Methods and Table S1 summarizes the cohort-specific genotyping, imputation, and quality control procedures, including the number of SNPs used in cohort-specific analyses (ranging from 1.9 million to 2.7 million).

\section{Power analysis}

We estimated that our sample size of 5,823 represented an effective sample size of 5,186 taking account of the non-independent observations in the family studies. With this sample size, we had $80 \%$ power to detect a genetic variant accounting for as little as $0.77 \%$ of the variance in $\mathrm{CAC}$ quantity with genome-wide significance and as little as $0.15 \%$ with nominal significance $(\mathrm{p}<0.05)$ (Figure 1 ). Our sample had $>80 \%$ power to detect a variant with comparable effect size to that in $9 \mathrm{p} 21$ associated with CAC in EA (effect size $=0.009$, or $0.9 \%$; unpublished data, 2012). Thus, our AA CAC study was adequately powered to detect effect sizes comparable to those observed for the top associated SNPs in the EA GWAS of CAC. However lower allele frequencies in African descent samples could lead to a lower overall effect size, even if the effect of the allele is the same as in European samples.

\section{Heritability analysis}

The heritability of CAC scores was estimated in each of the five family studies (Table 1 ). The range of heritability across these AA family samples (0-47\%) tended to be lower than those reported among EA CAC studies (40$60 \%$ ) [18-21]. The AA estimate in GeneSTAR (0\%) may be sensitive to the small sample size and lower prevalence of CAC relative to EA families, but it is consistent with a lower heritability of CAC in AA compared to EA. Setting aside the GeneSTAR study, we estimate the heritability of CAC in AA to be $\sim 30 \%$ which still suggests lower heritability in AA compared to EA.

\section{Meta-analysis findings}

The quantile-quantile (QQ) plot for the combined AA GWA meta-analysis is shown in Figure 2A. Principal components were used in each cohort-specific analysis and lambda values were between 0.96 and 1.1, thus no genomic control correction was applied to our results. 


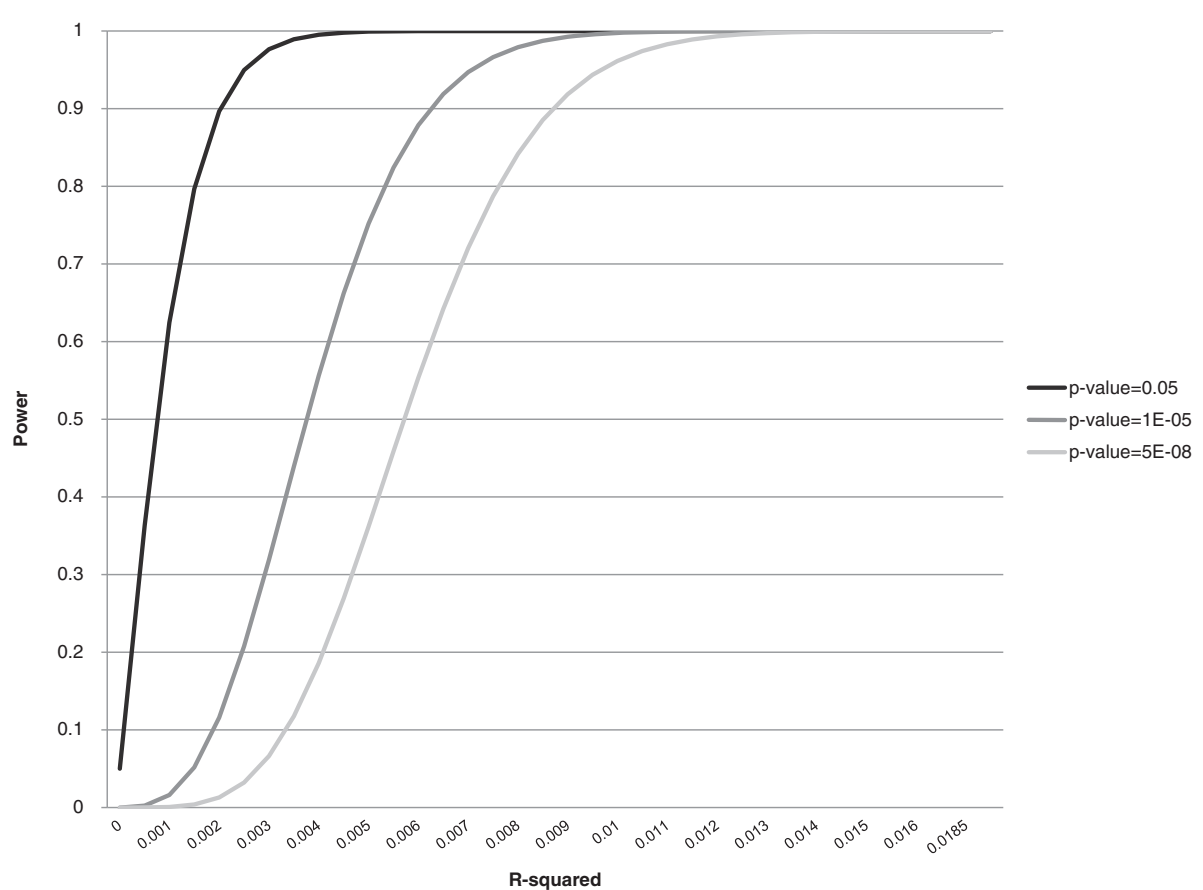

Figure 1 Power curves. Power curves calculated using QUANTO [32,33] software, as described in the text. In brief, we specified a quantitative outcome, assumed an additive genetic model and used an effective sample size of 5,186 with the estimated mean and standard deviation of $\ln (C A C+1)$. Allelic frequency variation did not affect the power estimates. We characterized the effect size as $r^{2}$.

QQ plots for cohort-specific GWA analyses are shown in Additional file 2: Figure S1. As summarized in Additional file 1: Table S2 and shown on the Manhattan plot in Figure 2B, our meta-analysis yielded no genome-wide significant results and there was limited evidence of clustering of top SNPs at a single chromosomal location. The SNP with the smallest $p$ value was rs749924 on chromosome 2, $\mathrm{p}=1.07 \mathrm{E}-07$. We focused on SNPs with $\mathrm{p}<1 \mathrm{E}-05$, which identified 67 SNPs with suggestive statistical evidence of association with CAC (Additional file 1: Table S2). These 67 SNPs represent 45 potentially independent signals (using SimpleM [38]) and included none of the loci associated with CAC in EA [22]. The secondary analyses produced similar results to the primary analysis and therefore these results are not presented.

\section{Evidence for association of suggestive AA SNPs in data from individuals of European ancestry}

We interrogated our 67 most significant SNPs (original or best proxy, using the Broad Institutes SNP Annotation and Proxy Search (SNAP) website; http://www.broadinstitute.
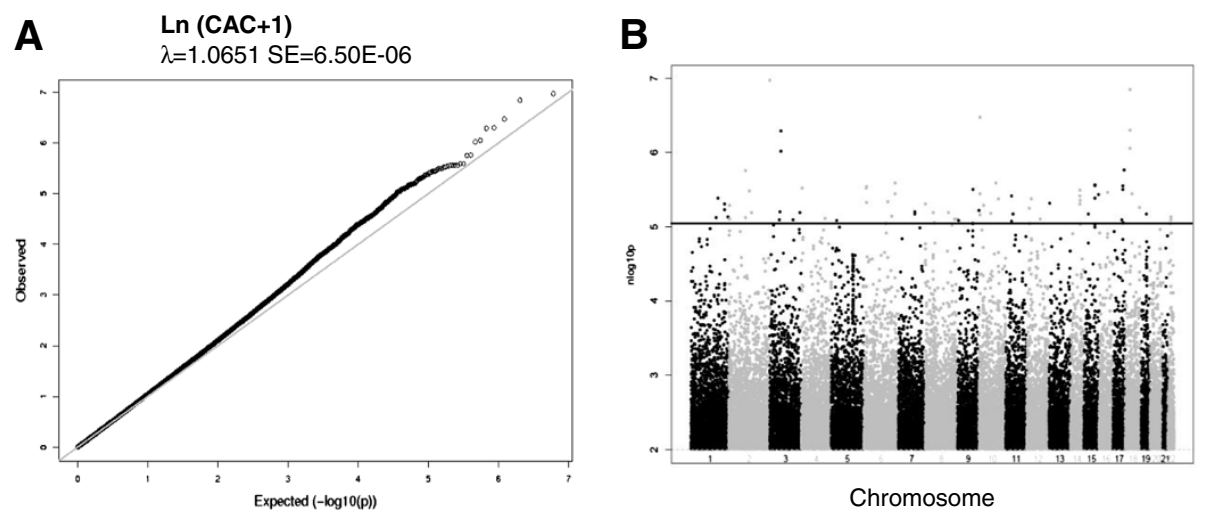

Figure 2 Quantile-quantile and Manhattan plots of AA CAC GWAS results. A) Quantile-quantile plot for the Meta-analysis of CAC. B) Manhattan plot for the meta-analysis of SNPs associated with CAC. No SNPs reach genome-wide significance, however SNPs above the blue line indicate the suggestive significance level of $p<1.0 \mathrm{E}-05$. 
org/mpg/snap/ldsearch.php) for their association with CAC in the CHARGE meta-analysis of individuals of EA [22]. Of these 67 SNPs with suggestive CAC association ( $\mathrm{p}<1 \mathrm{E}-05)$ in AA, three (one each in SOX9, PRKCA, and NRG1) reached nominal significance $(\mathrm{p} \leq 0.05)$ for association with $\ln (\mathrm{CAC}+1)$ in CHARGE EA data (Additional file 1: Table S2). However, 2 of the 3 SNPs (in SOX9 and PRKCA) showed association in an inconsistent allelic direction of effect and while rs1462872 in NRG1 demonstrated an association in the same direction, the $\mathrm{p}$ value in EA was 0.05 . Due to multiple testing comparisons and given this single isolated hit in a relatively large gene, we suspect this association is a false positive result, suggesting that there was no meaningful evidence for replication of these AA SNP signals in the CHARGE EA sample.

\section{Interrogation of reported EA CHD GWAS signals in this AA CAC GWAS}

We amassed EA CHD GWAS signals (for CAC and for CAD/MI e.g., with specific focus on the 9p21 region and PHACTR1 locus) to assess their signals in the AA CAC GWAS results.

\section{EA CAC GWAS signals in African Americans}

Recently, O'Donnell and colleagues [22] identified several SNPs that were genome-wide significant in two regions in a GWAS of CAC in EA. These regions, 9p21 and PHACTR1 on 6p24.1, are also associated with CAD/MI [2-6]. We queried these SNPs (or their proxies, identified using SNAP) for CAC association in AAs. Of 49 SNPs reaching genome-wide significance for CAC in EAs, $44(89.8 \%)$ had the same direction of effects on CAC in AAs. Six of these directionally consistent SNPs also had a p-value $<0.1$ in AA, which we consider nominally significant for a one-sided test suggesting modest enrichment for EA CAC alleles within our AA sample (EA CAC GWAS significant SNPs with replication $p$ values $<0.10$ in AA CAC meta-analysis shown in Table 2, results for all previously reported suggestive SNPs $(\mathrm{p}<1 \mathrm{E}-05)$ in EA CAC have AA CAC results reported in Additional file 1: Table S3).

\section{EA CAD GWAS signals in African Americans}

Since CAC is a strong indicator of risk for cardiovascular endpoints, we interrogated top genome-wide significant SNPs (or their proxies) from large GWAS metaanalyses in EA for CAD/MI [2-6] in our AA CAC results. Of 34 SNPs at previously identified loci for CAD/MI in EA [5], 25 (73.5\%) had the same direction of effects on CAC in AAs but none, including the top EA 9p21 SNP, were nominally significant in our AA data (Additional file 1: Table S4).

\section{Signals from the 9p21 fine mapping regions defined for EA and $A A$}

We queried the association in AA of 166 SNPs within the EA region for $C A D$ and $C A C$ in the 9 p21 region, and we identified 24 SNPs with nominal evidence for association $(\mathrm{p} \leq 0.05)$. The peak AA CAC association mapped to a different SNP than those reported in other populations and to a smaller linkage disequilibrium (LD) region reported in the CARe fine mapping effort for CHD in AA [11], with the strongest association at rs16905644 (CAF 0.11, $\mathrm{p}=4.07 \mathrm{E}-05$; Bonferroni correction for 166 SNPs tested, $\mathrm{p}=0.0068$; Figure 3 ). Overall, ten of these 24 nominally associated SNPs localized within this smaller AA region, but fourteen lay outside this AA region but still within the larger EA region (Additional file 1: Table S5). However, neither the strongest 9p21 EA SNPs for CAC (rs1333049) or CAD (rs4977574) in EA nor rs6475606 or rs3217989 at 9p21, recently reported to be associated with CHD in AA [40] were among these nominally significant signals (Figure 3 and Additional file 1: Table S5).

\section{Signals from the PHACTR1 locus for CAC and CAD in EA}

We queried the association in AA of approximately 20 SNPs within the $\mathrm{EA}$ region for $\mathrm{CAD}$ and $\mathrm{CAC}$ in PHACTR1 [3,5,22] and we identified no SNP with even nominal evidence for association $(\mathrm{p} \leq 0.05)$. However, of these 20 SNPs, the lead SNP from the EA CAC analysis, rs9349379, had a p-value of 0.09 in the AA CAC analysis with similar direction of effect, but the allele frequency was markedly different (CAF EA = 0.59; CAF AA =0.90). We further examined all SNPs within PHACTR1 (12.713.3 megabases) in the AA CAC GWAS, including a comparison of the LD structure of this region in EA and AA populations. The most significant PHACTR1 association in our AA CAC GWAS was an intronic SNP, rs7768030 ( $p=0.004$, Additional file 1: Table S7 for all results), which is located $80.9 \mathrm{~kb}$ from the lead EA CAC SNP, and even further from the lead EA MI SNP, rs12526453 [3,5]. The rs7768030 SNP is not in long range LD with these two EA SNPs, as evidenced from data from HapMap (Additional file 1: Table S8). Indeed, based on HapMap, there are marked differences between AA and EA LD patterns in this region (Additional file 3: Figure S2); we present the regional plots in EA and AA for the FamHS CAC data around these top hits in PHACTR1 (rs9349379 and rs7768030, respectively; Additional file 4: Figure S3). Overall, based on these analyses, there may be a signal for CAC in AA at PHACTR1 but in a different region of this large gene than that observed for CAC and CAD/MI in EA.

\section{Discussion}

Despite higher rates of $\mathrm{CHD}$ in AA [8], adequately powered genomic studies in this population are lacking. We leveraged data from almost all available US-based 
Table 2 CHARGe European ancestry CAC meta-analysis [22] SNP top hits with nominal significance in the African American CAC meta-analysis

\section{EA CHARGE meta-analysis results $(n=9,992) \quad$ AA CAC meta-analysis look-up $(n=5,823)$}

\begin{tabular}{|c|c|c|c|c|c|c|c|c|c|c|c|c|c|c|c|c|}
\hline \multirow[b]{2}{*}{ SNP } & \multirow[b]{2}{*}{ Chrom } & \multirow[b]{2}{*}{ Position } & \multirow[b]{2}{*}{$\begin{array}{l}\text { Closest } \\
\text { gene }\end{array}$} & \multirow[b]{2}{*}{ Rolet } & & \\
\hline & & & & & $\begin{array}{l}\text { Coded } \\
\text { allele }\end{array}$ & $\begin{array}{l}\text { Coded } \\
\text { allele } \\
\text { freq }\end{array}$ & Effect & SE‡ & $p \neq$ & $\begin{array}{l}\text { Direction of point estimate } \\
\text { for the association } \S\end{array}$ & $\begin{array}{l}\text { Coded } \\
\text { allele }\end{array}$ & $\begin{array}{l}\text { Coded } \\
\text { allele } \\
\text { freq }\end{array}$ & Effect & SE & $p$ & $\begin{array}{l}\text { Direction of point estimate } \\
\text { for the association } \S\end{array}$ \\
\hline rs3218020 & 9 & 21997872 & CDKN2A & & $A$ & 0.34 & 0.19 & 0.03 & $\begin{array}{l}2.53 \mathrm{E}- \\
09\end{array}$ & +++++ & A & 0.15 & 0.15 & 0.05 & 0.002 & +++-++-- \\
\hline rs1537375 & 9 & 22116071 & CDKN2B & & $\mathrm{T}$ & 0.50 & -0.24 & 0.03 & $\begin{array}{l}5.06 \mathrm{E}- \\
16\end{array}$ & ---- & $T$ & 0.33 & -0.08 & 0.04 & 0.03 & +------+ \\
\hline rs9349379 & 6 & 12903957 & PHACTR1 & intron & $A$ & 0.59 & -0.21 & 0.03 & $\begin{array}{l}2.65 \mathrm{E}- \\
11\end{array}$ & ---+- & $A$ & 0.90 & -0.14 & 0.08 & 0.07 & $-+?+--++$ \\
\hline rs4977575 & 9 & 22124744 & CDKN2B & & $C$ & 0.52 & -0.27 & 0.03 & $\begin{array}{l}9.93 \mathrm{E}- \\
19\end{array}$ & ----- & $C$ & 0.12 & -0.09 & 0.05 & 0.08 & ---+---+ \\
\hline rs1333042 & 9 & 22103813 & CDKN2B & & A & 0.51 & -0.24 & 0.03 & $\begin{array}{l}4.54 \mathrm{E}- \\
16\end{array}$ & ---- & $A$ & 0.12 & -0.09 & 0.05 & 0.09 & +---+--- \\
\hline rs10511701 & 9 & 22112599 & CDKN2B & & $\mathrm{T}$ & 0.49 & -0.24 & 0.03 & $\begin{array}{l}4.48 \mathrm{E}- \\
16\end{array}$ & ---- & $T$ & 0.28 & -0.07 & 0.04 & 0.09 & +---+--+ \\
\hline
\end{tabular}

tf no role indicated, then is outside known gene boundaries.

\#SE: Standard error; $p$ : $p$-value.

§order of studies: for EA CHARGE: Age, Gene/Environment Susceptibility Study-Reykjavik (AGES), Rotterdam Study-ll, Framingham Heart Study, GENOA, Rotterdam Study-l; and for AA CAC: FamHS, JHS, CARDIA, JHS-

ARIC, MESA, MESA Family/Air, GeneSTAR, GENOA. GWAS results from each study were completed independently, thus data availability varied by study depending on study specific imputation quality and genotyping

quality control for each SNP. Therefore not all studies had results for all SNPs. 


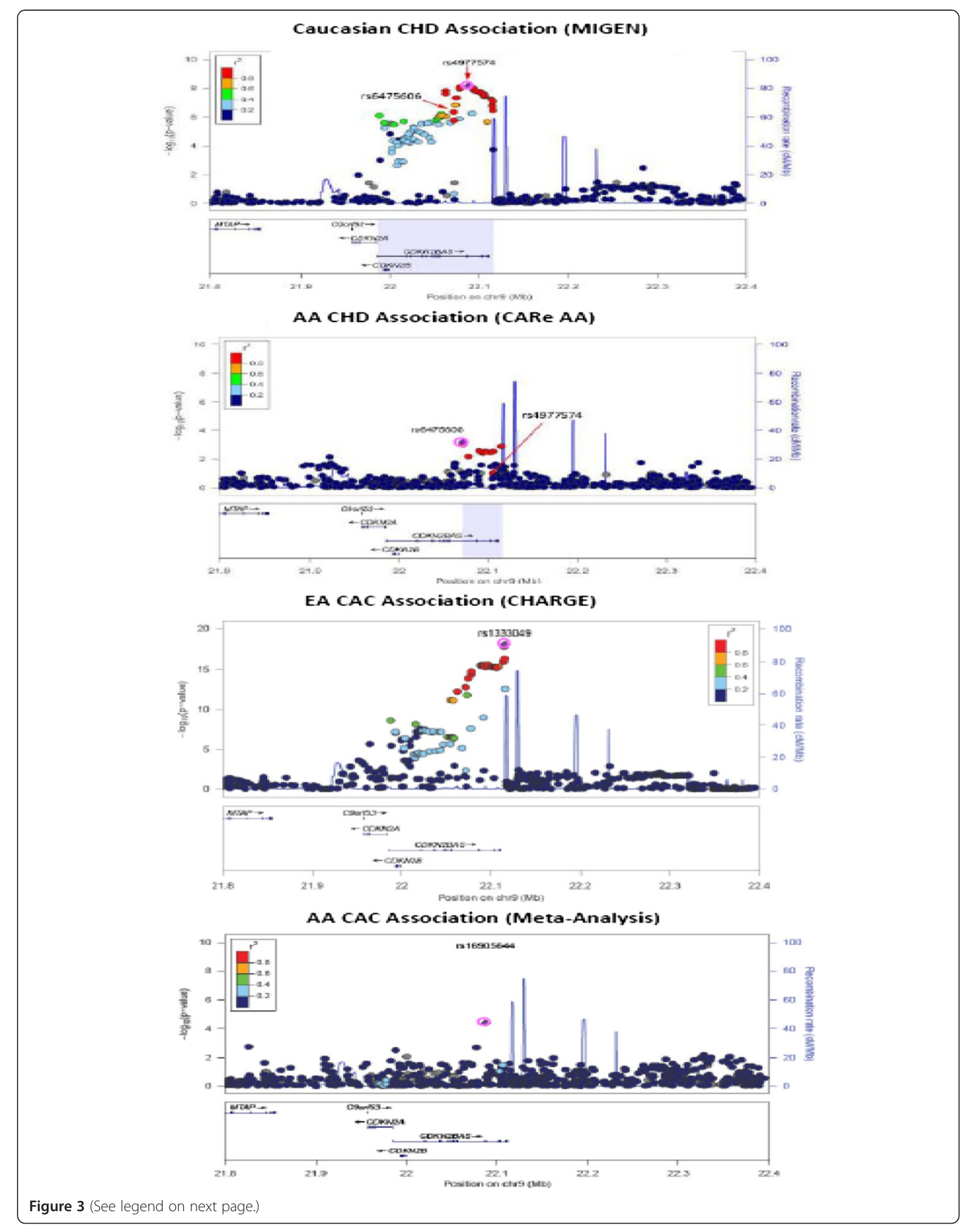


(See figure on previous page.)

Figure 3 LocusZoom plots of the 9p21 region. Fine mapping of the 9p21 region using LOCUS ZOOM [39]. The top plot uses data from MIGen [3] for the Caucasian CHD associations (lead EA SNP is rs4977574 on right and lead AA SNP from CARe CHD is rs6475606 on left). The second plot demonstrates the AA CHD associations, highlighting the same two SNPs as the first graph [11]. The third plot depicts the EA CAC associations [22] and its lead SNP, rs1333049. The final plot depicts our AA CAC associations with lead SNP rs16905644 (AA CAC meta-analysis associations for all these SNPs are in Additional file 1: Supplemental Table S5). In all plots, the region associated with EA is broader than the region associated with $\mathrm{AAs}$ and the top results for $\mathrm{AA}$ CAC are in the same region as that associated with CHD in $\mathrm{AA}$ [11].

studies of CAC in AAs and performed a meta-analysis of their GWAS results with the goal of identifying novel loci using this validated marker of subclinical coronary atherosclerosis and predictor of CHD. Using this largest collection of CAC in AAs, we observed substantial heritability for $\mathrm{CAC}$ in $\mathrm{AA}$, albeit lower than that reported for EA [18-21], and we failed to reveal loci for CAC at genome-wide significant levels despite having adequate power to detect common alleles with moderate to large effect sizes. We found no credible support for our top AA GWAS meta-analysis findings in the EA CHARGE CAC GWAS [22]. We note that we were not able to perform the ideal replication, which would be a separate analysis of CAC in an independent AA sample. The majority of genome wide significant loci identified for CAC in EA (49 SNPs in 2 gene regions) [22] and CAD/MI in EA (34 SNPs at 34 distinct regions) [2-6] had the same direction of allelic effect in our AA GWAS data but only four reached nominal significance $(\mathrm{p} \leq 0.05)$ with similar direction of effect. Because inconsistent findings between AA and EA might represent genetic differences between the populations, we interrogated SNP associations across the 9p21 region $[5,22,41]$ which has documented distinct LD structure among individuals of EA and individuals of AA [11] and SNP associations across PHACTR1 [22]. Indeed, in the 9p21 region we detected a SNP with suggestive evidence of an association with CAC in AA (rs16905644, p=4.07E-05) but within the smaller region of LD in AA. At the PHACTR1 locus, there were distinct patterns of LD and allele frequencies in Africans compared to Europeans with a weak association of PHACTR1 SNPs with CAC in AA at some distance from that for CAC and CAD/MI in EA, but still within the recognized gene boundaries. Although this is suggestive of a separate signal in PHACTR1 among AA, further fine mapping and interrogation of rare variants is required to determine if PHACTR1 is a bona fide locus for CAC in AA populations. Despite modest suggestive findings in AA at 9p21 and some other CAC and CAD EA loci, these data suggest that even larger samples than we analyzed with race specific fine mapping will be required for $\mathrm{CAC}$ GWAS discoveries in AA populations.

The lack of novel or strong confirmatory signals in our analysis of AA samples may be attributable to several factors. Although we had adequate power to detect effects similar to those observed for the strongest loci in
EA, it is possible that the actual marginal genetic effects in AAs are smaller. It seems unlikely, however, that this is the whole explanation for the apparent lack of overlap between EA and AA signals for CAC. One factor that may attenuate signals in AAs is the smaller haplotype blocks (decreased levels and range of LD across the genome) as compared with EA populations [5,22,41]. As a consequence, it is possible that the imputed reference panel of SNPs used in this study are inadequate tags of the AA genome with the consequence that we may miss functional SNP signals due to inadequate coverage. Lower LD between measured tag variants and unmeasured functional variants will result in a net decrease in effect size and, thus, lower power for detection. This concern can be addressed with denser, race-specific gene maps or sequencing in AA populations, but until then, we cannot verify the relevance of EA variants in AAs.

Another factor that may affect our ability to detect trait loci relates to racial differences in the heritability of the CAC trait. We estimated the heritability of CAC in several participating AA family studies, and found up to $47 \%$ of the CAC variance to be influenced by the genetic variation, with a lower bound estimate of zero for one study. Setting aside this study with $0 \%$ heritability, the heritability of CAC in AA is $\sim 30 \%$ which is lower than that reported in EA samples ( 50\%) [18-21]. Heritability estimates may reflect population differences between AA and EA samples in known risk factors for CAC, but comparison between race, within studies (e.g., in FamHS $33 \%$ for AA vs. $45 \%$ for EA, North et al. [21]; in GeneSTAR $0 \%$ for AA vs. 27\% for EA) reinforces the possibility of differences between AA and EA in the impact of genetic variation on CAC. This apparent lower level of CAC heritability in AAs could reflect a relatively greater importance of non-genetic factors and gene by environment interactions as compared to additive genetic effects [42].

It is also possible that different biological pathways or different genetic variants within the same pathways are at play resulting in genetic heterogeneity between European and African ancestral populations in the mechanisms leading to atherogenesis and CAC. This is borne out by the observation that greater CAC burden is associated with higher levels of European admixture in AA populations [43], suggesting that genetic variants specific to EA play a more important role in the development of CAC than 
those of African origin. Indeed, there are several lines of evidence suggesting distinct pathophysiology of CAC in $\mathrm{AAs}$, including lower $\mathrm{CAC}$ scores despite greater risk factor burden and higher rates of CHD in AA samples $[7,9,10,23,24,26,44-46]$. Therefore, while some EA variants may play a role in atherosclerosis in AA, other distinct pathways may be important. In this case, validation in EA populations, as we attempted, would not be expected to succeed. Finally, environmental factors, either by themselves or interacting with genetic background, may have a more prominent role in CAC and atherosclerosis in AA than genetic effects. CAC scores do, however, predict CHD events in AA [16] samples suggesting that larger studies pursuing genetic discoveries using CAC in AA should provide some insights into mechanisms and risk of CHD in this population.

Our study has several strengths. First, it is the largest GWAS of any sub-clinical atherosclerosis trait in AAs. Second, it leverages data from nearly all AA cardiovascular cohort studies and represents a cross-section of the US AA population. An attribute of our study was the $a$ priori planning such that GWAS datasets were analyzed using raw data from the cohorts in a pre-specified manner rather than a post-hoc combination of results, followed by attempted validation of our top findings in EA GWAS CAC datasets. Third, we used multiple family datasets to obtain heritability estimates of CAC in AA. Finally, although the results of this work are largely negative, it highlights the need to pursue additional genetic epidemiological studies of CHD in AA populations.

Our study also has several limitations. Although our sample was the largest GWAS of a sub-clinical atherosclerosis trait in AAs and powered for loci with comparable effect sizes to the strongest loci identified in EA GWAS, this study was underpowered to discover SNPs with small effects. We lacked a positive control genotype that could support the power of our study to detect expected genetic effects for CAD; PCSK9 and LPA genotypes associated with CAD in AA were not genotyped and lacked proxy SNPs in our data. However, we did interrogate the well-documented 9p21 and PHACTR1 regions, including the known different LD structure in the 9 p21 region $[5,22,41]$ as a potential positive control; indeed, this 9p21 analysis, using an appropriate ethnicspecific LD focus, did provide suggestive/nominal evidence for 9p21 locus effects on CAC in AA; and weak evidence for PHACTR1 locus effects on CAC in AA.

\section{Conclusion}

In summary, our results for the largest AA CAC GWAS amassed to date are remarkable in two respects: first, in the lack of support in EA data for the top signals arising from AA data and second, in the weak support for association of EA CAC [22] and CAD and MI loci [2-6] in our AA sample. Substantial biological differences in the genomic architecture of $\mathrm{CAC}$, atherosclerosis and clinical $\mathrm{CHD}$ between AA and EA populations are likely.

\section{Additional files}

\begin{abstract}
Additional file 1: Supplemental methods and Table S1. Cohort-
specific genotyping, imputation, and quality control procedures/criteria. Table S2. AA CAC meta-analysis SNP 'top hits' and their assessment in the CHARGe EA CAC meta-analysis [12]. Table S3. Assessment in AfricanAmericans of SNPs previously associated with CAC in the CHARGe EA CAC Meta-Analysis [12]. Table S4. Assessment in African-Americans of loci previously associated with coronary artery disease. Table S5. SNP signals within EA and AA LD blocks at the 9p21 region as defined by CARe AA CHD GWAS*. Table S6. Participant characteristics of the CHARGE EA CAC sample [12]. Table S7. Assessment of SNP associations in PHACTR1 region in the AA CAC Meta-Analysis. Table S8. Attributes of top PHACTR1 SNPs from O'Donnell ${ }^{\dagger}$, CARDIoGRAM $^{*}$, MIGEN $^{\S}$, and AA $\mathrm{CAC}^{\ddagger}$ obtained from HapMap.
\end{abstract}

Additional file 2: Figure S1. Quantile-quantile plots of AA CAC GWAS results from each study.

Additional file 3: Figure S2. Linkage disequilibrium plots from HapMap. A) For the CEPH population and B) For the YRI population, both for the region of PHACTR1 from $12800 \mathrm{~kb}-13100 \mathrm{~kb}$. Blue arrow points to lead EA CAC SNP from O'Donnell et al, rs9349379; purple arrow points to AA CAC meta-analysis lead SNP in PHACTR1, rs7768030; green arrow is rs2026458 from O'Donnell et al; orange arrow is rs12526453 from MIGEN and CardioGRAM. As depicted, there is vastly different LD structure between these populations and these SNPs are in different LD blocks.

Additional file 4: Figure S3. Regional plots of association results for the region from 12.7 Mb - 13.3 Mb in PHACTR1. This uses A) EA CAC data from FamHS (in house data) and B) AA CAC meta-analysis results. There is little LD between the two top hits, rs9349379 in EA and rs7768030 in AA (purple diamonds in figure); however they may be tagging some common underlying functional variant that is not genotyped.

\section{Abbreviations}

CHD: Coronary heart disease; CAC: Coronary artery calcification; AA: African Americans; SNP: Single nucleotide polymorphism; EA: European ancestry; PHACTR1: Phosphatase and actin regulator 1; GWAS: Genome-wide association study; CAD: Coronary artery disease; MI: Myocardial infarction; NHLBI: National heart, lung, and blood institute; CARe: Candidate gene association resource; FamHS: Family heart study; JHS: Jackson heart study; ARIC: Atherosclerosis risk in communities study; JHS-ARIC: Jackson heart study-atherosclerosis risk in communities study; CARDIA: Coronary artery risk development in young adults; MESA: Multi-ethnic study of atherosclerosis; GeneSTAR: Genetic study of atherosclerosis risk; GENOA: Genetic epidemiology network of arteriopathy; CT: Computed tomography; CAF: Coded allele frequency; QQ: Quantile-quantile plot; HWE: HardyWeinberg equilibrium; SOLAR: Sequential oligogenic linkage analysis routines; SE: Standard error; SNAP: SNP annotation and proxy search; P: p-value; NRG1: Neuregulin 1; SOX9: SRY (sex determining region Y)-box 9; PRKCA: Protein kinase C, alpha; LD: Linkage disequilibrium; PCSK9: Proprotein convertase subtilisin/kexin type 9; LPA: Lipoprotein, Lp(a); n: Sample size; Q1: $25^{\text {th }}$ percentile; Q3: $75^{\text {th }}$ percentile; Chrom: Chromosome.

\section{Competing interests}

The authors do not have any conflicts of interest, financial or otherwise.

\section{Authors' contributions}

NDW, LAL, THM, XG, SKG, SRH, MEG, DHO, MB, JJC, HAT, DAB, SD, DNP, JFP, BMP, DMB, MAP, WSP, CJO, JGW, TBH, MK, LAC, JIR, MF, LCB, PAP, IBB, and MPR conceived and designed the study. MKW, ML, LFB, KFK, APR, LRY, LQ, $L A L$, JFF, JH, TY, JAS, BGK, XG, QW, S-JH, BMP, DMB, MAP, WSP, CJO, JGW, $T B H, L A C, M F, J I R, L C B, P A P, I B B$, and MPR acquired the data. MKW, ML, LFB, KFK, APR, LRY, LQ, CCW, LAL, JFF, JH, TY, JAS, BGK, XG, QW, MB, JJC, S-JH, BMP, DMB, MAP, WSP, CJO, JGW, TBH, LAC, JIR, MF, LCB, and PAP analyzed the data. MKW, ML, LFB, KFK, APR, NDW, LRY, LQ, LAL, JFF, JH, THM, JAS, BGK, 
XG, QW, SKG, SRH, MEG, DHO, MB, JJC, HAT, DAB, SD, S-JH, DNP, JFP, BMP, DMB, MAP, WSP, CJO, JGW, TBH, MK, LAC, JIR, MF, LCB, PAP, IBB, and MPR interpreted the data. MKW, IBB, and MPR drafted the manuscript. All authors revised the manuscript for important intellectual content, read, and approved the final manuscript.

\section{Authors' information}

Ingrid B. Borecki and Muredach P. Reilly are senior authors.

\section{Acknowledgements}

The authors acknowledge the essential role of all the participating cohorts: NHLBI Family Heart Study, all cohorts (JHS, JHS-ARIC, CARDIA, and MESA) involved in the NHLBI Candidate gene Association Resource (CARe) Consortium studies, MESA Family/Air, GeneSTAR, and GENOA. The collaboration of the CHARGE EA CAC consortia also played a vital role.

\section{Sources of funding}

The National Heart, Lung, and Blood Institute's Family Heart Study (FamHS) was supported by NIH grants R01-HL-087700 and R01-HL-088215 (Michael A. Province, PI) from NHLBl; and R01-DK-8925601 and R01-DK075681 (Ingrid B. Borecki, PI) from NIDDK.

The authors from the CARe Consortium wish to acknowledge the support of the National Heart, Lung, and Blood Institute and the contributions of the research institutions, study investigators, field staff and study participants in creating this resource for biomedical research. The following studies have contributed parent study data, ancillary study data, and DNA samples through the Broad Institute (N01-HC-65226).

Coronary Artery Risk in Young Adults (CARDIA): University of Alabama at Birmingham (N01-HC-48047), University of Minnesota (N01-HC-48048), Northwestern University (N01-HC-48049), Kaiser Foundation Research Institute (N01-HC-48050), University of Alabama at Birmingham (N01-HC95095), Tufts-New England Medical Center (N01-HC-45204), Wake Forest University (N01-HC-45205), Harbor-UCLA Research and Education Institute (N01-HC-05187), University of California, Irvine (N01-HC-45134, NO1-HC95100); Jackson Heart Study (JHS): Jackson State University (N01-HC-95170), University of Mississippi (N01-HC-95171), Tougaloo College (N01-HC-95172); Multi-Ethnic Study of Atherosclerosis (MESA): University of Washington (N01-HC-95159),Regents of the University of California (N01-HC-95160), Columbia University (N01-HC-95161), Johns Hopkins University (NO1-HC95162, N01-HC-95168), University of Minnesota (N01-HC-95163), Northwestern University (NO1-HC-95164), Wake Forest University (N01-HC95165), University of Vermont (N01-HC-95166), New England Medical Center (N01-HC-95167), Harbor-UCLA Research and Education Institute (N01-HC95169), Cedars-Sinai Medical Center (R01-HL-071205), University of Virginia (subcontract to R01-HL-071205).

The Coronary Artery Risk Development in Young Adults (CARDIA) study is funded by contracts N01-HC-95095, N01-HC-48047, N01-HC-48048, N01-HC48049, N01-HC-48050, N01-HC-45134, N01-HC-05187, N01-HC-45205, and N01-HC-45204 from the National Heart, Lung, and Blood Institute to the CARDIA investigators. GWAS genotyping and quality control for the CARDIA African-Americans was supported by the NHLBI's Candidate-gene Association REsource (CARe) Study. Statistical analysis of CARDIA data was supported by grants R01-HL084099 and U01-HG004729 to MF. This manuscript has been reviewed by CARDIA for scientific content and consistency of data interpretation with previous CARDIA publications.

The MESA Family/Air Studies were conducted and supported by the National Heart, Lung, and Blood Institute (NHLBI) and the United States Environmental Protection Agency (EPA) in collaboration with MESA Family and MESA Air investigators, respectively. Support for MESA Family is provided by grants and contracts R01HL071051, R01HL071205, R01HL071250, R01HL071251, R01HL071252, R01HL071258, and R01HL071259. Support for MESA Air is provided by grant RD83169701. Funding for genotyping was provided by NHLBI Contract N02-HL-6-4278. Genotyping was performed at the Broad Institute of Harvard and MIT (Boston, Massachusetts, USA) and at Affymetrix (Santa Clara, California, USA) using the Affymetrix Genome-Wide Human SNP Array 6.0.

The GeneSTAR Study was supported by the National Heart, Lung, and Blood Institute (NHLBI) through the STAMPEED (R01 HL087698-01) consortium as well as grants HL58625-01A1, HL59684, and HL071025-01A1, and a grant from the NIH/National Institute of Nursing Research (NR008153-01). Additional support was provided by a grant from the $\mathrm{NIH} / \mathrm{National}$ Center for Research Resources (M01-RR000052) to the Johns Hopkins General Clinical Research Center.

The Genetic Epidemiology Network of Arteriopathy (GENOA) is supported by the National Institutes of Health, grant numbers HL085571, HL087660, and HL100245 from National Heart, Lung, Blood Institute. We thank Eric Boerwinkle, PhD from the Human Genetics Center and Institute of Molecular Medicine and Division of Epidemiology, University of Texas Health Science Center, Houston, Texas, USA and Julie Cunningham, PhD from the Department of Health Sciences Research, Mayo Clinic College of Medicine, Rochester, MN, USA for their help with genotyping.

MPR is supported by R01-DK071224, R01-DK-090505, U01-HL108636, K24HL107643 and R01-HL113147.

MK is supported by the AXA Research Fund.

\section{Author details}

'Department of Genetics, Division of Statistical Genomics, Washington University School of Medicine, St. Louis, MO, USA. 'Biostatistics and Epidemiology, University of Pennsylvania, Philadelphia, PA, USA. ${ }^{3}$ Department of Epidemiology, University of Michigan School of Public Health, Ann Arbor, MI, USA. ${ }^{4}$ Department of Biostatistics, University of Washington, Seattle, WA, USA. ${ }^{5}$ Department of Epidemiology, University of Washington, Seattle, WA, USA. ${ }^{6}$ Heart Disease Prevention Program, University of California, Irvine, CA, USA. ${ }^{7}$ Department of Medicine, The Johns Hopkins University School of Medicine, Baltimore, MD, USA. ${ }^{8}$ Department of Biostatistics, Boston University School of Public Health, Boston, MA, USA. ${ }^{9}$ Department of Genetics, University of North Carolina, Chapel Hill, NC, USA. ${ }^{10}$ The Cardiovascular Institute and Department of Medicine, Perelman School of Medicine, University of Pennsylvania, Philadelphia, PA, USA. "11Program in Medical and Population Genetics, Broad Institute, Cambridge, MA, USA. ${ }^{12}$ Department of Medicine, University of Mississippi Medical Center, Jackson, MS, USA. ${ }^{13}$ Institute for Translational Genomics and Population Sciences, Los Angeles Biomedical Research Institute at Harbor-UCLA Medical Center, Torrance, CA, USA. ${ }^{14}$ Division of Cardiovascular Medicine, Department of Internal Medicine, University of Michigan, Ann Arbor, MI, USA. ${ }^{15}$ Center of Biostatistics and Bioinformatics, University of Mississippi Medical Center, Jackson, MS, USA. ${ }^{16}$ Department of Radiology, Tufts University School of Medicine, Boston, MA, USA. ${ }^{17}$ University of California Los Angeles School of Medicine, Los Angeles, CA, USA. ${ }^{18}$ Department of Radiology, Wake Forest University School of Medicine, Winston-Salem, NC, USA. ${ }^{19}$ Jackson State University, Tougaloo College, Jackson, MS, USA. ${ }^{20}$ The University of Mississippi Medical Center, Jackson, MS, USA. ${ }^{21}$ Radiology and Imaging Sciences, National Institutes of Health Clinical Center, Bethesda, MD, USA. ${ }^{22}$ National Heart, Lung, and Blood Institute's Framingham Heart Study and the Center for Population Studies, Framingham, MA, USA. ${ }^{23}$ Advanced Technologies and Surgery Branch, Division of Cardiovascular Sciences, National Heart, Lung, and Blood Institute, National Institutes of Health, Bethesda, MD, USA. ${ }^{24}$ Cardiovascular Health Research Unit, Departments of Medicine, Epidemiology, and Health Service, University of Washington, Seattle, WA, USA. ${ }^{25}$ Group Health Research Institute, Group Health Cooperative, Seattle, WA, USA. ${ }^{26}$ Departments of Epidemiology and Medicine, The Johns Hopkins School of Medicine and Public Health, Baltimore, MD, USA. ${ }^{27}$ National Heart, Lung, and Blood Institute's Framingham Heart Study, Framingham, MA, USA. ${ }^{28}$ Cardiology Division, Department of Medicine, Massachusetts General Hospital, Harvard Medical School, Boston, MA, USA. ${ }^{29}$ National Heart, Lung, and Blood Institute, Bethesda, MD, USA. ${ }^{30}$ Department of Physiology and Biophysics, University of Mississippi, Jackson, MS, USA. ${ }^{31}$ Laboratory of Epidemiology, Demography, and Biometry, Intramural Research Program, National Institute on Aging, National Institutes of Health, Bethesda, MD, USA. ${ }^{32}$ Netherlands GenomicsInitiative-Sponsored Netherlands Consortium for Healthy Aging, Rotterdam, The Netherlands. ${ }^{33}$ Department of Epidemiology, Erasmus University Medical Center, Rotterdam, The Netherlands. ${ }^{34}$ Department of Biostatistics, Boston University School of Public Health, Boston, MA, USA. ${ }^{35}$ Houston Institute of Molecular Medicine, University of Texas, Houston, TX, USA.

Received: 12 December 2012 Accepted: 18 July 2013 Published: 19 July 2013

\section{References}

1. NHLBI: Morbidity and Mortality: 2009 Chart Book on Cardiovascular, Lung, and Blood Diseases. Bethesda, MD: National Institutes of Health; 2009. 
2. Samani NJ, Erdmann J, Hall AS, Hengstenberg C, Mangino M, Mayer B, Dixon RJ, Meitinger T, Braund P, Wichmann HE, et al: Genomewide association analysis of coronary artery disease. N Engl J Med 2007, 357(5):443-453.

3. Kathiresan S, Voight BF, Purcell S, Musunuru K, Ardissino D, Mannucci PM, Anand S, Engert JC, Samani NJ, Schunkert H, et al: Genome-wide association of early-onset myocardial infarction with single nucleotide polymorphisms and copy number variants. Nat Genet 2009, 41(3):334-341.

4. Reilly MP, Li M, He J, Ferguson JF, Stylianou IM, Mehta NN, Burnett MS, Devaney JM, Knouff CW, Thompson JR, et al: Identification of ADAMTS7 as a novel locus for coronary atherosclerosis and association of $A B O$ with myocardial infarction in the presence of coronary atherosclerosis: two genome-wide association studies. Lancet 2011, 377(9763):383-392.

5. Schunkert H, Konig IR, Kathiresan S, Reilly MP, Assimes TL, Holm H, Preuss M, Stewart AF, Barbalic M, Gieger C, et al: Large-scale association analysis identifies 13 new susceptibility loci for coronary artery disease. Nat Genet 2011, 43(4):333-338.

6. Coronary Artery Disease (C4D) Genetics Consortium: A genome-wide association study in Europeans and South Asians identifies five new loci for coronary artery disease. Nat Genet 2011, 43(4):339-344.

7. Mensah GA, Mokdad AH, Ford ES, Greenlund KJ, Croft JB: State of disparities in cardiovascular health in the United States. Circulation 2005, 111(10):1233-1241.

8. Doherty TM, Tang W, Detrano RC: Racial differences in the significance of coronary calcium in asymptomatic black and white subjects with coronary risk factors. J Am Coll Cardiol 1999, 34(3):787-794.

9. Clark LT, Ferdinand KC, Flack JM, Gavin JR 3rd, Hall WD, Kumanyika SK, Reed JW, Saunders E, Valantine HA, Watson K, et al: Coronary heart disease in African Americans. Heart Dis 2001, 3(2):97-108.

10. Wong MD, Shapiro MF, Boscardin WJ, Ettner SL: Contribution of major diseases to disparities in mortality. N Engl J Med 2002, 347(20):1585-1592.

11. Lettre $G$, Palmer CD, Young T, Ejebe KG, Allayee $H$, Benjamin EJ, Bennett $F$, Bowden DW, Chakravarti A, Dreisbach A, et al: Genome-wide association study of coronary heart disease and its risk factors in 8,090 African Americans: the NHLBI CARe Project. PLoS Genet 2011, 7(2):e1001300.

12. Cohen JC, Boerwinkle $E_{1}$ Mosley TH Jr, Hobbs HH: Sequence variations in PCSK9, low LDL, and protection against coronary heart disease. N Engl J Med 2006, 354(12):1264-1272.

13. Cohen J, Pertsemlidis A, Kotowski IK, Graham R, Garcia CK, Hobbs HH: Low $\mathrm{LDL}$ cholesterol in individuals of African descent resulting from frequent nonsense mutations in PCSK9. Nat Genet 2005, 37(2):161-165.

14. Guerci $A D$, Arad $Y$, Agatston A: Predictive value of EBCT scanning. Circulation 1998, 97(25):2583-2584.

15. Bielak LF, Rumberger JA, Sheedy PF 2nd, Schwartz RS, Peyser PA: Probabilistic model for prediction of angiographically defined obstructive coronary artery disease using electron beam computed tomography calcium score strata. Circulation 2000, 102(4):380-385.

16. Detrano R, Guerci AD, Carr JJ, Bild DE, Burke G, Folsom AR, Liu K, Shea S, Szklo $M$, Bluemke DA, et al: Coronary calcium as a predictor of coronary events in four racial or ethnic groups. N Engl J Med 2008, 358(13):1336-1345.

17. Greenland $\mathrm{P}$, Bonow RO, Brundage BH, Budoff $M J$, Eisenberg MJ, Grundy SM, Lauer MS, Post WS, Raggi P, Redberg RF, et al: ACCF/AHA 2007 clinical expert consensus document on coronary artery calcium scoring by computed tomography in global cardiovascular risk assessment and in evaluation of patients with chest pain: a report of the American college of cardiology foundation clinical expert consensus task force (ACCF/AHA writing committee to update the 2000 expert consensus document on electron beam computed tomography). Circulation 2007, 115(3):402-426.

18. Peyser PA, Bielak LF, Chu JS, Turner ST, Ellsworth DL, Boerwinkle E, Sheedy PF 2nd: Heritability of coronary artery calcium quantity measured by electron beam computed tomography in asymptomatic adults. Circulation 2002, 106(3):304-308.

19. Fischer M, Broeckel U, Holmer S, Baessler A, Hengstenberg C, Mayer B, Erdmann J, Klein G, Riegger G, Jacob HJ, et al: Distinct heritable patterns of angiographic coronary artery disease in families with myocardial infarction. Circulation 2005, 111(7):855-862.

20. Rampersaud E, Bielak LF, Parsa A, Shen H, Post W, Ryan KA, Donnelly P, Rumberger JA, Sheedy PF 2nd, Peyser PA, et al: The association of coronary artery calcification and carotid artery intima-media thickness with distinct, traditional coronary artery disease risk factors in asymptomatic adults. Am J Epidemiol 2008, 168(9):1016-1023.
21. North KE, Carr JJ, Borecki IB, Kraja A, Province M, Pankow JS, Wilk JB, Hixson JE, Heiss G: QTL-specific genotype-by-smoking interaction and burden of calcified coronary atherosclerosis: the NHLBI Family heart study. Atherosclerosis 2007, 193(1):11-19.

22. O'Donnell CJ, Kavousi M, Smith AV, Kardia SL, Feitosa MF, Hwang SJ, Sun Y, Province MA, Aspelund T, Dehghan A, et al: Genome-wide association study for coronary artery calcification with follow-up in myocardial infarction. Circulation 2011, 124(25):2855-2864.

23. Lee TC, O'Malley PG, Feuerstein I, Taylor AJ: The prevalence and severity of coronary artery calcification on coronary artery computed tomography in black and white subjects. J Am Coll Cardiol 2003, 41(1):39-44.

24. Bild DE, Detrano R, Peterson D, Guerci A, Liu K, Shahar E, Ouyang P, Jackson S, Saad MF: Ethnic differences in coronary calcification: the multi-ethnic study of Atherosclerosis (MESA). Circulation 2005, 111(10):1313-1320.

25. Kawakubo M, LaBree L, Xiang M, Doherty TM, Wong ND, Azen S, Detrano R: Race-ethnic differences in the extent, prevalence, and progression of coronary calcium. Ethn Dis 2005, 15(2):198-204.

26. Budoff MJ, Nasir K, Mao S, Tseng PH, Chau A, Liu ST, Flores F, Blumenthal RS: Ethnic differences of the presence and severity of coronary atherosclerosis. Atherosclerosis 2006, 187(2):343-350.

27. Musunuru K, Lettre G, Young T, Farlow DN, Pirruccello JP, Ejebe KG, Keating BJ, Yang Q, Chen MH, Lapchyk N, et al: Candidate gene association resource (CARe): design, methods, and proof of concept. Circ Cardiovasc Genet 2010, 3(3):267-275.

28. Budoff MJ, Katz R, Wong ND, Nasir K, Mao SS, Takasu J, Kronmal R, Detrano $\mathrm{RC}$, Shavelle DM, Blumenthal RS, et al: Effect of scanner type on the reproducibility of extracoronary measures of calcification: the multiethnic study of atherosclerosis. Acad Radiol 2007, 14(9):1043-1049.

29. Carr JJ, Danitschek JA, Goff DC, Crouse JR 3rd, D'Agostino R, Chen MY, Burke $\mathrm{GL}$ : Coronary artery calcium quantification with retrospectively gated helical CT: protocols and techniques. Int J Cardiovasc Imaging 2001, 17(3):213-220.

30. Carr JJ, Nelson JC, Wong ND, MCNitt-Gray M, Arad Y, Jacobs DR Jr, Sidney S, Bild DE, Williams OD, Detrano RC: Calcified coronary artery plaque measurement with cardiac CT in population-based studies: standardized protocol of multi-ethnic study of Atherosclerosis (MESA) and coronary artery risk development in young adults (CARDIA) study. Radiology 2005 , 234(1):35-43.

31. Agatston AS, Janowitz WR, Hildner FJ, Zusmer NR, Viamonte M Jr, Detrano R: Quantification of coronary artery calcium using ultrafast computed tomography. J Am Coll Cardiol 1990, 15(4):827-832.

32. Gauderman W: Candidate gene association analysis for a quantitative trait, using parent-offspring trios. Genet Epidemiol 2003, 25:327-338.

33. Gauderman W, Morrison J: QUANTO 1.1: A computer program for power and sample size calculations for genetic-epidemiology studies. 2006. http://hydra.usc.edu/gxe.

34. Li Y, Willer CJ, Ding J, Scheet $P$, Abecasis GR: MaCH: using sequence and genotype data to estimate haplotypes and unobserved genotypes. Genet Epidemiol 2010, 34(8):816-834.

35. Howie BN, Donnelly P, Marchini J: A flexible and accurate genotype imputation method for the next generation of genome-wide association studies. PLoS Genet 2009, 5(6):e1000529.

36. Price AL, Patterson NJ, Plenge RM, Weinblatt ME, Shadick NA, Reich D: Principal components analysis corrects for stratification in genome-wide association studies. Nat Genet 2006, 38(8):904-909.

37. Almasy $L$, Blangero J: Multipoint quantitative-trait linkage analysis in general pedigrees. Am J Hum Genet 1998, 62(5):1198-1211.

38. Gao X, Starmer J, Martin ER: A multiple testing correction method for genetic association studies using correlated single nucleotide polymorphisms. Genet Epidemiol 2008, 32(4):361-369.

39. Pruim RJ, Welch RP, Sanna S, Teslovich TM, Chines PS, Gliedt TP, Boehnke M, Abecasis GR, Willer CJ: LocusZoom: regional visualization of genome-wide association scan results. Bioinformatics 2010, 26(18):2336-2337.

40. Kral BG, Mathias RA, Suktitipat B, Ruczinski I, Vaidya D, Yanek LR, Quyyumi AA, Patel RS, Zafari AM, Vaccarino $V$, et al: A common variant in the CDKN2B gene on chromosome $9 \mathrm{p} 21$ protects against coronary artery disease in Americans of African ancestry. J Hum Genet 2011, 56(3):224-229.

41. Musunuru K, Kathiresan S: Genetics of coronary artery disease. Annu Rev Genomics Hum Genet 2010, 11:91-108.

42. Diez Roux AV, Detrano R, Jackson S, Jacobs DR Jr, Schreiner PJ, Shea S, Szklo $\mathrm{M}$ : Acculturation and socioeconomic position as predictors of coronary calcification in a multiethnic sample. Circulation 2005, 112(11):1557-1565. 
43. Wassel CL, Pankow JS, Peralta CA, Choudhry S, Seldin MF, Arnett DK: Genetic ancestry is associated with subclinical cardiovascular disease in African-Americans and Hispanics from the multi-ethnic study of atherosclerosis. Circ Cardiovasc Genet 2009, 2(6):629-636.

44. Orakzai SH, Orakzai RH, Nasir K, Santos RD, Edmundowicz D, Budoff MJ, Blumenthal RS: Subclinical coronary atherosclerosis: racial profiling is necessary. Am Heart J 2006, 152(5):819-827.

45. Howard BV, Criqui MH, Curb JD, Rodabough R, Safford MM, Santoro N, Wilson AC, Wylie-Rosett J: Risk factor clustering in the insulin resistance syndrome and its relationship to cardiovascular disease in postmenopausal white, black, hispanic, and Asian/Pacific Islander women. Metab Clin Exp 2003, 52(3):362-371.

46. Kronmal RA, McClelland RL, Detrano R, Shea S, Lima JA, Cushman M, Bild $D E$, Burke GL: Risk factors for the progression of coronary artery calcification in asymptomatic subjects: results from the multi-ethnic study of Atherosclerosis (MESA). Circulation 2007, 115(21):2722-2730.

doi:10.1186/1471-2350-14-75

Cite this article as: Wojczynski et al:: Genetics of coronary artery

calcification among African Americans, a meta-analysis. BMC Medical Genetics 2013 14:75

\section{Submit your next manuscript to BioMed Central and take full advantage of:}

- Convenient online submission

- Thorough peer review

- No space constraints or color figure charges

- Immediate publication on acceptance

- Inclusion in PubMed, CAS, Scopus and Google Scholar

- Research which is freely available for redistribution 